\title{
Design and Manufacturing of a Novel Shear Thickening Fluid Composite (STFC) with Enhanced out-of-Plane Properties and Damage Suppression
}

\author{
F. Pinto ${ }^{1} \cdot$ M. Meo ${ }^{1}$
}

Received: 12 September 2016 / Accepted: 14 September 2016 / Published online: 27 September 2016

(C) The Author(s) 2016. This article is published with open access at Springerlink.com

\begin{abstract}
The ability to absorb a large amount of energy during an impact event without generating critical damages represents a key feature of new generation composite systems. Indeed, the intrinsic layered nature of composite materials allows the embodiment of specific hybrid plies within the stacking sequence that can be exploited to increase impact resistance and damping of the entire structure without dramatic weight increase. This work is based on the development of an impact-resistant hybrid composite obtained by including a thin layer of Non-Newtonian silica based fluid in a carbon fibres reinforced polymer (CFRP) laminate. This hybrid phase is able to respond to an external solicitation by activating an order-disorder transition that thickens the fluid increasing its viscosity, hence dissipating the energy impact without any critical failure. Several Shear Thickening Fluids (STFs) were manufactured by changing the dimensions of the particles that constitute the disperse phase and their concentrations into the continuous phase. The dynamic viscosity of the different STFs was evaluated via rheometric tests, observing both shear thinning and shear thickening effects depending on the concentration of silica particles. The solutions were then embedded as an active layer within the stacking sequence to manufacture the hybrid CFRP laminates with different embedded STFs. Free vibration tests were carried out in order to assess the damping properties of the different laminates, while low velocity impact tests were used to evaluate their impact properties. Results indicate that the presence of the non-Newtonian fluid is able to absorb up to $45 \%$ of the energy during an impact event for impacts at $2.5 \mathrm{~m} / \mathrm{s}$ depending on the different concentrations and particles dimensions. These results were confirmed via C-Scan analyses to assess the extent of the internal delamination.
\end{abstract}

Keywords Delamination · Shear thicknening fluid · Impact damage

\section{Meo}

m.meo@bath.ac.uk

1 Department of Mechanical Engineering, University of Bath, Bath BA2 7AY, UK 


\section{Introduction}

One of the most important aspects that concerns all the structural materials and, more in particular composite structures, is constituted by the behavioural uncertainties associated with impacts with foreign objects. Indeed, because of their weak resistance to through-the-thickness solicitations, these materials are susceptible to delamination damages under impact loads that can compromise their integrity and lead to catastrophic failures. Based on this premise, multifunctional composite systems play a fundamental role in the development of new generation impact resistant materials due to the possibility to modify their specific properties by adding or modifying specific phases within the laminate structure. Indeed, by following this approach, it is possible to enhance the impact properties of a traditional laminate by manufacturing a Shear Thickening Fluid (STF) that can subsequently be included within the laminate sequence as an active layer for impact damage suppression.

STFs are non-Newtonian fluids with remarkable properties that behave like a liquid when there are no forces applied and turn in a very stiff solid-like structure in presence of high shear rates [1]. Since this liquid-solid transition is the result of a rapid increase of viscosity, these highly nonlinear fluids have been exploited for the design of damping systems and shock absorbers.

Liquid body armour based on the inclusion of STF have been studied intensively during the last decade [2-5], and several commercial products (mostly reinforced soft protections based generally on aramid fibres) are available on the market. However, very few works have been done on the design of stiff composite materials with embedded STF (without the use of aramid woven fabric). In the work carried out by Fisher et al., an STF was sandwiched between polyvinyl chloride beams, showing good results in damping the vibrations of the entire structure [6], while Soutrenon et al. realised a structural damper by embedding the STF within an open-cell foam encapsulated in silicone [7]. Another multifunctional system patented by Picken [8] is based on the preparation of an STF using a supramolecular polymer (polymers made of monomers held together by noncovalent interactions [9]) that is then used to impregnate the fibres and produce a composite material. The main focus of this work is the development a engineered STF that is can be embedded directly as an active layer within the fibrous reinforcement of a composite laminate in order to manufacture stiff CFRP parts characterised by higher levels of energy absorption due to the thickening of the solution during impacts with foreign objects. The technique does not imply any modifications of the thermoset resin used for the composite manufacturing, therefore the procedure can be carried out using commercial thermoset/carbon fibres prepregs.

\section{Theoretical Aspects}

Generally, a non-Newtonian fluid can be defined as a material whose viscosity is dependent on the shear rate or shear rate history. However, included in the group there are some fluids in which it is possible to observe normal stress-differences or other non-Newtonian behaviour even if they are characterised by shear-independent viscosity.

Figure 1 shows the rheology of different kinds of Non Newtonian fluids, according to the different relationships between shear stress and shear rate.

A Shear Thickening Fluid (see red curve in the figure above) is a non-Newtonian fluid obtained by dispersing spherical small particles (dispersed phase) within a liquid phase (continuous phase). Increasing the particles' concentration beyond dilute limit leads to the 
Fig. 1 Rheology of nonNewtonian fluids

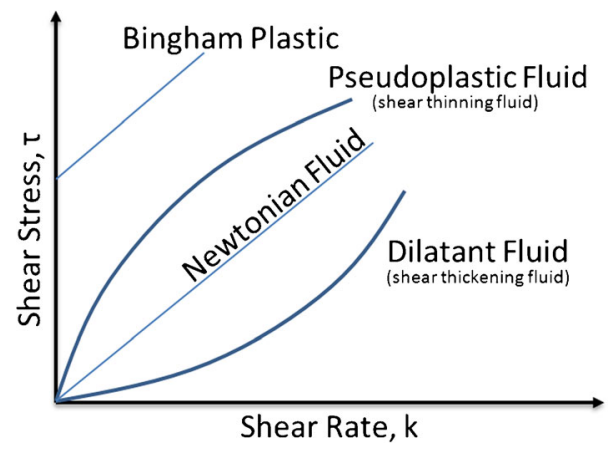

activation of particle-particle interactions and the creation of long range order, resulting in nonNewtonian effects such as shear thinning or shear thickening. These effects can be explained with transitions from an ordered to a disordered state that occur when high stresses are applied [10]. Indeed, when this fluid is subjected to a relatively low shear rate, the repulsive interactions between particles are able to create an ordered and layered structure that consents a more effective particles flow through the liquid phase, reducing the viscosity of the solution. At this point, if the shear rate is increased above a critical point $\left(\gamma_{\mathrm{c}}\right)$, the particle-particle interactions are overcome by shear forces and the particles are squeezed together, moving the fluid out from its equilibrium state and resulting in a disordered and more complex structure. In particular, shear thickening occurs when the repulsive Brownian forces are overcome by attractive hydrodynamic shear forces, leading to the formation and percolation of shear induced transient aggregates called "hydroclusters" that thickens the fluid [11].

As the hydroclusters are made of particles momentarily compressed together to form agglomerates, the particles' ability to flow around each other is hindered and requires more energy, leading to an abrupt increase in viscosity [12, 13]. When the shear stress is removed, the hydroclusters spread apart and the particles are separated, forming again a stable suspension (Fig. 2).

In order to observe shear-thickening behaviour in a suspension, there are three important factors to consider:

- Increasing the concentration of particles within the fluid will lead to an enhancement of the thickening effect, therefore the weight fraction has to be evaluated according to the system viscosity requirement [14]. For our purposes the concentration required is

Fig. 2 Schematics of Shear Thinning and Shear Thickening Effect

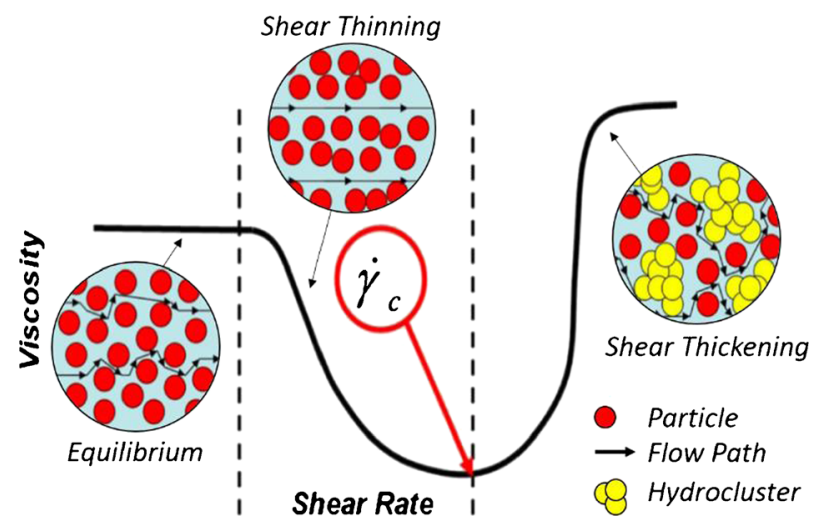


around 20-25\% wt. as it gives good results in terms of impact resistance and workability for composite applications. Further increase of the particles concentration leads to a very high level of initial viscosity of the solution (which is also dependent on the molecular weight of the continuous phase), decreasing its workability during the embedding process within the laminate. This problem can be partially solved by increasing the temperature during the manufacturing process. Moreover, volume fraction is strictly connected with particles dimensions as it can change the electrostatic interactions, leading to different forces interaction in the liquid/particles system.

- Suspension must not flocculate (destabilisation of the suspension): particles have to be neutral or repel one another by electrostatic, steric or entropic interactions. To achieve this, the continuous phase must induce steric or electrosteric stabilisation in order to enhance the colloid stability. Several carriers can be used for the preparation of the STF, such as ethylene glycol $[15,16]$, water [17] and other organic solvents, however the most used one for structural application is polyethylene glycol due to its tendency to form very stable suspension $[4,18]$ coupled with its biocompatibility.

- Particle volume fraction at which shear thickening behaviour is first observed is affected by the particle ratio. Studies present in literature report thickening behaviour observed for particles with a dimension range from $1 \mathrm{~nm}$ to $5000 \mathrm{~nm}$ [19]. Another important effect is given from the shape of the dispersed phase, however, in case of irregular particles, it is more difficult to understand the energy dispersion mechanism due to the superimposition of the thickening effect and the reciprocal friction between the inclusions [20].

Based on these considerations, the STF realised for this study forms a complex system which is characterised by a double change in its microstructure with the increase of the applied shear rate. Indeed, at low shear rate, the particles are rearranged from the equilibrium and become more organised in the flow, decreasing the fluid viscosity (shear thinning). A further increase of the shear rate leads to the formation of the hydroclusters and, consequently, to the thickening effect.

As for the particles used, there are several examples in literature of the different materials that can be employed, such as $\mathrm{CaCO}_{3}$ [20], PMMA [21, 22], PVC [23], PS and $\mathrm{SiO}_{2}$ [6, 13, 20]. For our purposes, different solutions were produced using two types of commercially available $\mathrm{SiO}_{2}$ with average diameters of 7 and $14 \mathrm{~nm}$ (Sigma Aldrich). Good thickening effects have been obtained for particles with a nominal diameter of $200 \mathrm{~nm}$.

\section{Experimental: Solution Preparing}

The components needed for manufacturing an STF are nanosized particles and a liquid carrier. For this work, several solutions were produced by mixing an increasing percentage of silica $\left(\mathrm{SiO}_{2}\right)$ particles (from 10 to $25 \%$ wt.) with PolyEthylene Glycol (HO- $\mathrm{CH}_{2}-\left(\mathrm{CH}_{2}-\mathrm{O}-\mathrm{CH}_{2}-\right)^{-}$$\left.\mathrm{CH}_{2}-\mathrm{OH}\right)$.

To ensure a good distribution of the silica within the carrier and to avoid the formation of agglomerates, an ultrasonic cavitation mixing method was used. Small quantities of silica particles were gradually added to the PEG and stirred in manually. Once a viscous gel was 
formed, the ultrasonic probe was immersed in gel and activated for $10 \mathrm{~min}$. High amplitude compressive waves generate localised low-pressure zones in the carrier, ensuring homogenous mixing of silica and PEG. At the end of the process the solution were stored under vacuum for $12 \mathrm{~h}$ in order to remove air bubbles and further improve the dispersibility.

\section{Rheological Behaviour of STF Solutions}

By following the approach of Stickel et al. [24] it is possible to define the viscosity of a suspension $(\eta)$ as a general function of multiple parameters which are based on the characteristics of the dispersed phase (the particles, indicated with the subscript $d p$ ) and the continuous phase (the suspending media, indicated with the subscript $c p$ ):

$$
\eta=f\left(r_{d p}, \rho_{d p}, C_{d p}, \rho_{c p}, \eta, k_{B} T, \dot{\gamma}, t\right)
$$

In particular, $r_{d p}, \rho_{d p}$ and $C_{d p}$ represent the hydrodynamic radius (which is equal to the particle radius in case of spherical particles), density and number concentration of the particles, while $\rho_{c p}, \eta_{c p}$ refer to density and viscosity of the suspending media. The additional terms that affects the viscosity are the thermal energy $\left(k_{B} T\right.$, where $k_{B}$ is the Boltzmann's constant and T is the absolute temperature), the shear rate $(\gamma)$ and time $(\mathrm{t})$. These variables can be reduced from eight to five by grouping the properties of the two phases in dimensionless quantities, leading to the definition of the following parameters:

$$
\begin{gathered}
\eta_{r}=\frac{\eta}{\eta_{c p}} \\
\rho_{r} \frac{\rho_{d p}}{\rho_{c p}} \\
\phi=\left(\frac{3}{4} \pi r_{d p}^{3}\right) C_{d p} \\
t_{r}=\frac{t k_{B} T}{\eta_{c p} r_{d p}^{3}}
\end{gathered}
$$

This reduces Eq. 1 to:

$$
\eta_{r}=f\left(\phi, \rho_{r}, k_{B} T, \dot{\gamma}, t_{r}\right)
$$

where $\phi$ represents the volume fractions of the particles within the solution. 
In order to further reduce the variables that influence the viscosity it is possible to introduce the Reynolds number for a spherical particle immersed in a fluid:

$$
\operatorname{Re}_{\dot{\gamma}}=\frac{\rho_{c p} v_{d p} r_{d p}}{\eta_{c p}}=\frac{\rho_{c p} r_{d p}^{2} \dot{\gamma}}{\eta_{c p}}
$$

Considering that the particles dimensions are below one micron, it is possible to assume very low values of the Reynolds number for the solution under study. As a consequence, if we consider a single particle of the dispersed phase, it is possible to correlate its diffusion through the continuous phase with the thermal energy and its mobility $\mu$ (the ratio of the particle's terminal drift velocity to an applied force) by following the Stokes-Einstein-Sutherland fluctuation-dissipation relationship, as discussed by Wagner et al. [10]:

$$
D=\mu k_{B} T=\frac{k_{B} T}{6 \pi \eta_{c p} r_{d p}}
$$

where the Stokes' law was used to express the mobility as the inverse of the drag coefficient $\left(\mu=\frac{1}{\zeta}=1 / 6 \pi \eta_{c p} r_{d p}\right)$.

From this expression, it is possible to estimate the time required for a particle to diffuse through a distance equal to its hydrodynamic radius as $r_{d p}^{2} / D_{0}$, from which high and low shear rates $\gamma$ can be defined.

The diffusion rate $D$ measures the interaction between the particles and the solvent molecules and can be related to the stress rate $\tau$ or the the shear rate $\gamma$ introducing the dimensionless Peclét number (Pe):

$$
P e_{\dot{\gamma}}=\frac{\tau r_{d p}^{3} 6 \pi \eta_{c p}}{k_{B} T}=\frac{\dot{\gamma} r_{d p}^{3}}{D_{0}}
$$

Using the expressions of the Reynolds and Peclét numbers, it is possible to rewrite the expression of the viscosity as:

$$
\eta_{r}=f\left(\phi, \rho_{r}, R e_{\dot{\gamma}}, P e_{\dot{\gamma}}, t_{r}\right)
$$

In addition, by considering that the particles of the dispersed phase are neutrally buoyant, it is possible to neglect both $\rho_{r}$ and $t_{r}$. Leading to:

$$
\eta_{r}=f\left(\phi, R e_{\dot{\gamma}}, P e_{\dot{\gamma}}\right)
$$

Since it correlates the shear rate with the rheology of a solution, the Peclét number can be used to analyse the behaviour of Non-Newtonian fluids. Indeed, while for low shear rates $\left(P e_{j}<1\right)$ the Brownian motion has the time to restore the fluid structure keeping it in an equilibrium state, when the shear rate increases, the fluid deformation operates in a shorter time scale than the Brownian motion, moving the solution out of its equilibrium state. 
Following Eq. 3, it appears clear that in order to investigate the properties of the STF, it is important to control two different parameters: the volume fraction of particles $(\phi)$ and the shear rate $(\dot{\gamma})$. Based on these considerations, several solutions were prepared with an increasing percentage of silica and the flow properties were measured using a Bohlin C-VOR Rheometer in parallel plate geometry (PP40) increasing the shear rate.

The results obtained from the rheometric analyses are shown in Fig. 3 and are quite revealing in several ways for all the tested solutions. First, it is clearly shown from the curves that both thinning and thickening effects are present for highly concentrated solutions, while for lower concentration the presence of secondary flows prevents accurate measurements of the weak thickening effect. Indeed, after an initial equilibrium state in which the fluid behaves almost like a Newtonian fluid, it is possible to observe a slight trend of decreasing viscosity for relatively low shear rates, while, when higher rates of shear are applied, the viscosity reaches a critical point in which the magnitudes of repulsive inter-particles forces are balanced by shear forces. When the shear stress exceeds this critical level, the shear forces that push the particles together become larger than the repulsive interactions so that the particles are moved from their equilibrium state, disrupting the ordered structure and leading to an abrupt increase of viscosity that thickens the fluid. Since the concentration of hydroclusters within the continuous phase is strictly related on the particles concentration [24], increasing the silica volume fraction within the STF makes the nonlinear effects more evident, enhancing thinning/thickening behaviours (see Fig. 4).

Initial viscosity of the solution depends strongly also on the molecular weight of the carrier. In this case the carrier used was a PEG $200 \mathrm{~g} \mathrm{~mol}^{-1}$. Results in terms of viscosity change due to thickening effects (two orders of magnitude increase) are not dissimilar to the ones found in literature [25].

Another important result can be observed from Fig. 5, which illustrates the existence of a strong relationship between volume fraction and the critical shear rate. Indeed, for higher particle concentrations, $\gamma_{\mathrm{c}}$ moves to lower values due to the reduced intra-particles distance, anticipating the transition between thinning and thickening effects, as reported by Maranzano et al. [14].

Fig. 3 Rheological analysis for STF at different concentrations: a) $\mathrm{STF} / 15$; b) STF/20; c) STF/25; d) comparison between the different curves

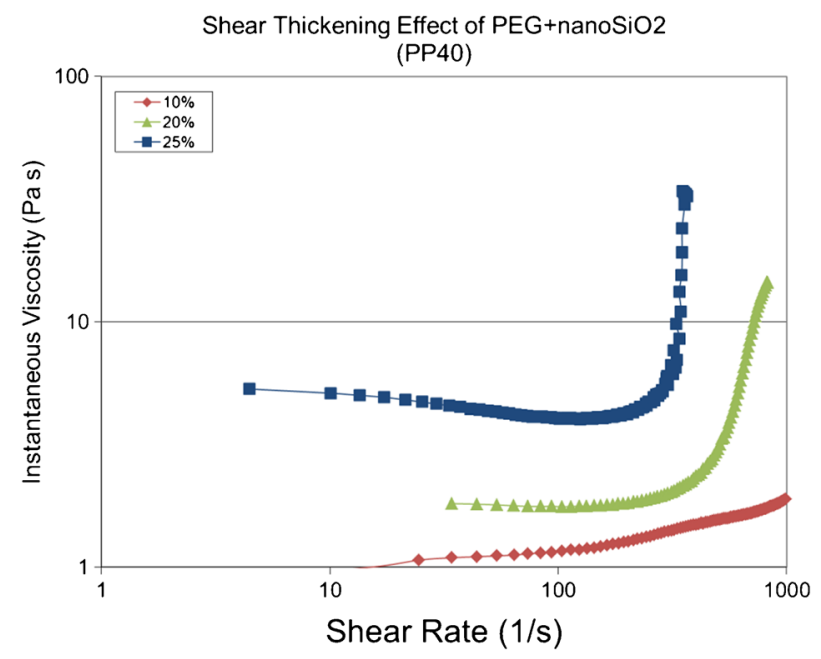


Increase with viscosity due to nanoparticles concentration

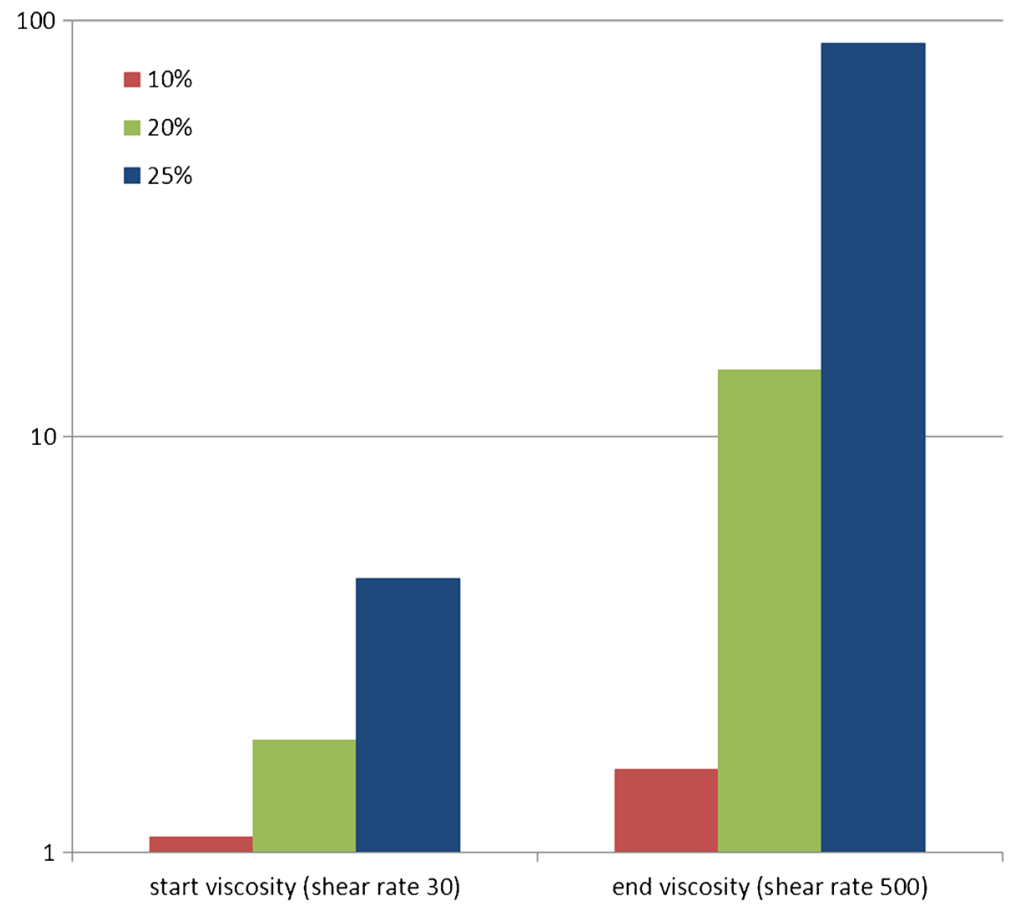

Fig. 4 viscosity increase with increasing silica concentration

\section{Composite Mechanical Characterisation}

The structural composite material was manufactured sandwiching a thin layer of STF between several layers of Carbon Fabric prepregs (see Fig. 6). The amount of fluid used in every sample has been kept constant for all the tests undertaken ( $10 \mathrm{~g}$ for $300 \mathrm{x} 150 \mathrm{~mm}$ plates), therefore we will refer to "STF percentage" as for the amount of silica within the fluid. Moreover, because of the small amount of STF involved in the hybridisation process, the total weight of the final laminate is not affected. Samples were prepared via Vacuum Assisted stratification followed

Fig. 5 Behaviour of critical shear rate with increasing particles concentration

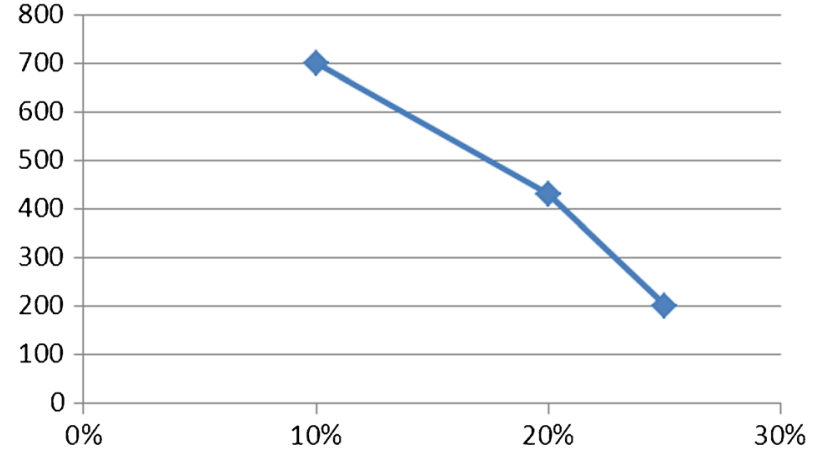




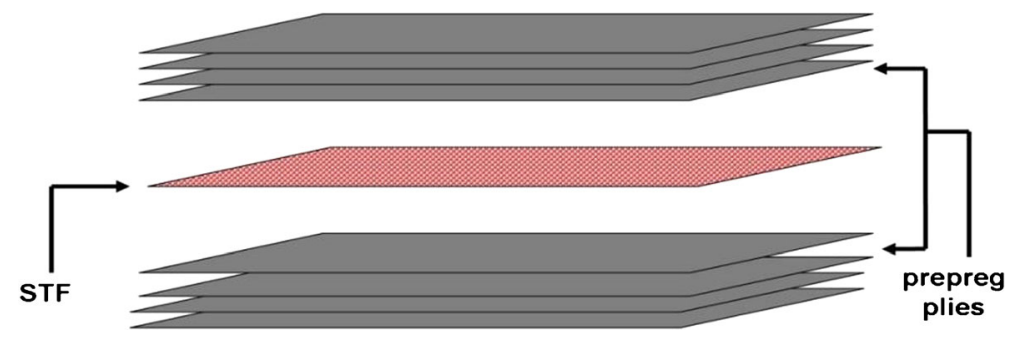

Fig. 6 Schematics of STF/CFRP composite preparation

by Autoclave consolidation in order to activate the curing reaction of the epoxy resin, and cut in $100 \times 150 \mathrm{~mm}$ plates for impact testing (Fig. 7).

\subsection{Damping Analysis}

In order to fully understand how the material would behave when subjected to an impact, experiments were conducted to evaluate the energy absorption of the STF/CFRP composite and a comparison was made with a traditional laminate, obtained with the same prepreg and the same lay-up sequence. A first series of test was undertaken to analyse the behaviour of the samples when subjected to a forced vibration using $\mathrm{n}$ Macro Fibre Composite (MFC) piezoelectric actuator to input a chirp signal over a $0-10 \mathrm{kHz}$ range of frequencies. Fast Fourier Transform (FFT) was performed in order to obtain the frequency content of the acquired vibration signals and evaluate the resonance frequencies of the samples.

The resonance frequencies of a structure are proportional to its stiffness, according to the relation $f_{i} \propto \sqrt{k / m}$, therefore it is possible to evaluate this mechanical characteristic by comparing the relative shift of frequencies peak patterns of hybridised samples and traditional unreinforced laminates [26]. Moreover, since the relative amplitude of the resonance peaks is a measurement of the ability of the material to store energy when subjected to an external load, it is possible to have an estimation of the damping ability of the structure by analysing its variation between the hybridised samples and the control ones.

Results from the tests are illustrated in Fig. 8 which clearly shows a visible shift to higher frequencies between the control sample and the hybridised one, indicating an increase in stiffness for the STF sample. Similar results have been observed for CNT reinforced composite structures [27]. In addition, the shifting of the amplitude of the entire frequency spectrum to lower levels suggests an enhancement of the damping behaviour of the structure due to the dynamic change in viscosity of the embedded STF.
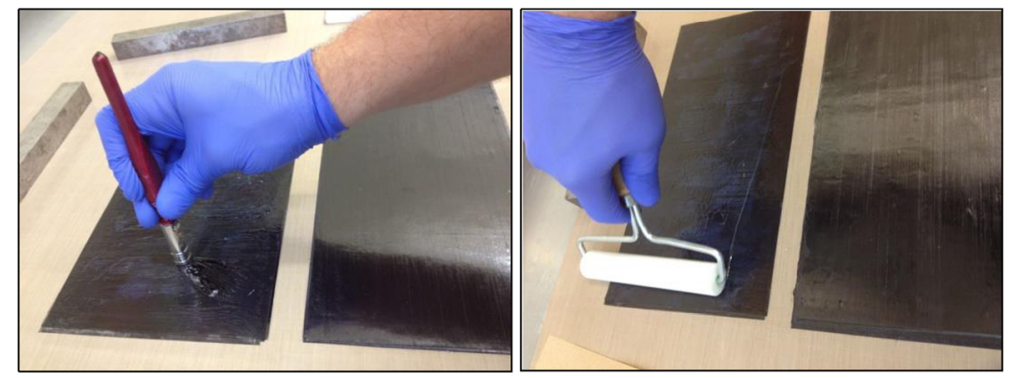

Fig. 7 manufacturing procedure of STF/CFRP composite samples 


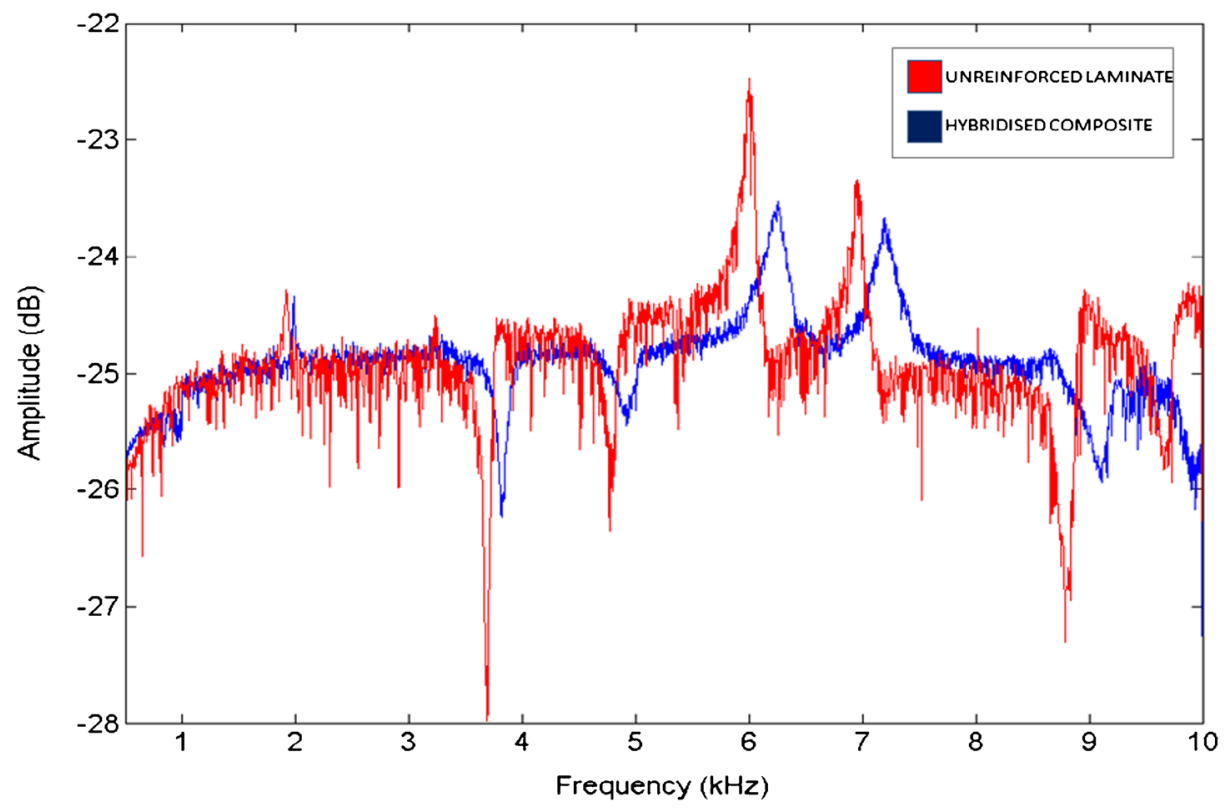

Fig. 8 Forced vibration signals for STF and Neat samples

A more in depth analysis on the damping behaviour of the STF based composites was carried out with free vibration tests in order to quantify the dissipation effect of the hybrid phase. Samples were tested in single cantilever beam geometry, clamping them to an optical bench and vibrations signals were collected using piezoelectric sensors.

Logarithmic decrement $\delta$ was evaluated from the acquired signals, following Eq. 4:

$$
\delta=\frac{1}{n} \ln \frac{x(t)}{x(t+n T)}
$$

Where $x(t)$ is the amplitude at time $t$, and $x(t+n T)$ is the amplitude calculated for a peak $n$ periods away. Subsequently, results obtained can be used to evaluate the damping ratio, by using Eq. 5:

$$
\zeta=\frac{1}{\sqrt{1+\left(\frac{2 \pi}{\delta}\right)^{2}}}
$$

Results from this series of tests demonstrate the effectiveness of the STF within the CFRP structure as structural damper (see Fig. 9). The trends of both logarithmic decrement and damping ratio $(+50 \%$ for both $\delta$ and $\zeta)$, clearly indicate how the inclusion of STF changes the damping properties of the material. This difference can be explained by the dynamic microstructure modification of the non-Newtonian fluid in the composite structure. Indeed, when the composite is subjected to an impact, part of the stress is transferred to the embedded STF and it activates the transient formation of hydroclusters. The abrupt increase in viscosity enhances the energy dissipation rate for the entire structure. 

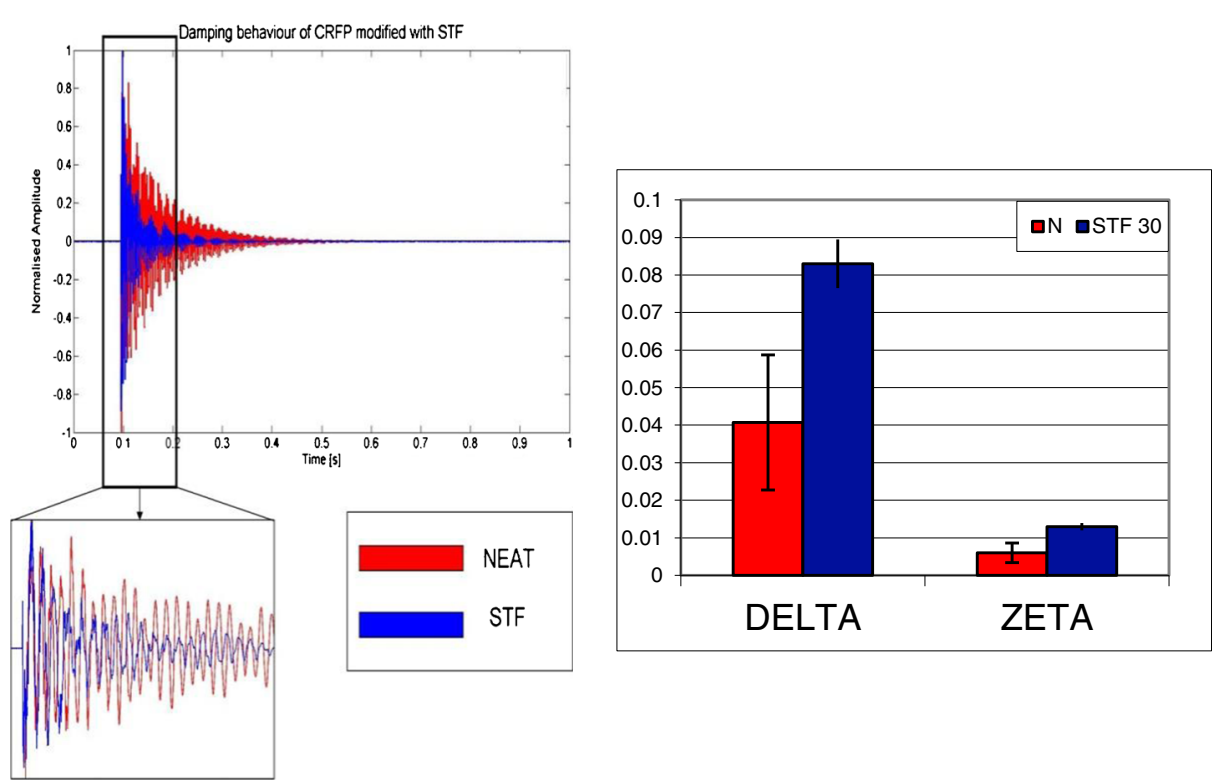

Fig. 9 Free vibration signals and results for STF and unreinforced samples

\subsection{Impact Testing}

In order to fully characterise the impact properties of the hybridised composite, several test were conducted on STF reinforced samples and a comparison was made with traditional laminates obtained with the same prepreg and the same layup procedure. Two different variables have been taken in account during these tests: silica particles dimensions ( 7 and $14 \mathrm{~nm}$ ) and particles concentration within the STF (from 10 to $25 \%$ ).

Tests were conducted using a drop tower rig with an impactor mass of $12,684 \mathrm{~kg}$ and a semi-spherical head geometry (20 mm diameter). Impacts were undertaken at 6, 20 and $40 \mathrm{~J}$ with velocities of $0.8,1.7$ and $2.5 \mathrm{~m} / \mathrm{s}$.

Figure 10 shows the curves obtained with the same impact energy for samples with increasing $7 \mathrm{~nm}$ particles concentration within the STF (herein referred as STF7/XX where XX stands for the silica concentration),

Results are summarised in Fig. 11 in which it is possible to observe that for low velocity impacts $(6 \mathrm{~J})$ the peak force stays linear (or within the experimental error) showing no large variations between control and reinforced samples.

For higher velocity impacts the effects of the hybridisation process becomes more consistent and affects the value of both force peak and the energy absorption rate.

Indeed, for the $20 \mathrm{~J}$ impacts, the presence of the STF leads to a slight increase $(\sim 12 \%)$ in the value of the force peaks, going from $4252 \mathrm{~N}$ (unreinforced samples) to $4724 \mathrm{~N}$ (STF25 samples), while there is no variation in the energy absorption rate.

The effects of the hybridisation are more evident for the $40 \mathrm{~J}$ impacts, where the curves show a dramatic increase in the force peaks, going from $5197 \mathrm{~N}$ for the unreinforced samples to a maximum of $6929 \mathrm{~N}$ for the STF based ones $(\sim 30 \%)$. It is possible to observe a relationship between this increment and the concentration of silica particles within the solution, as the force peaks values are enhanced by $12 \%$ for the STF15 and by an additional $20 \%$ for STF 25 . 


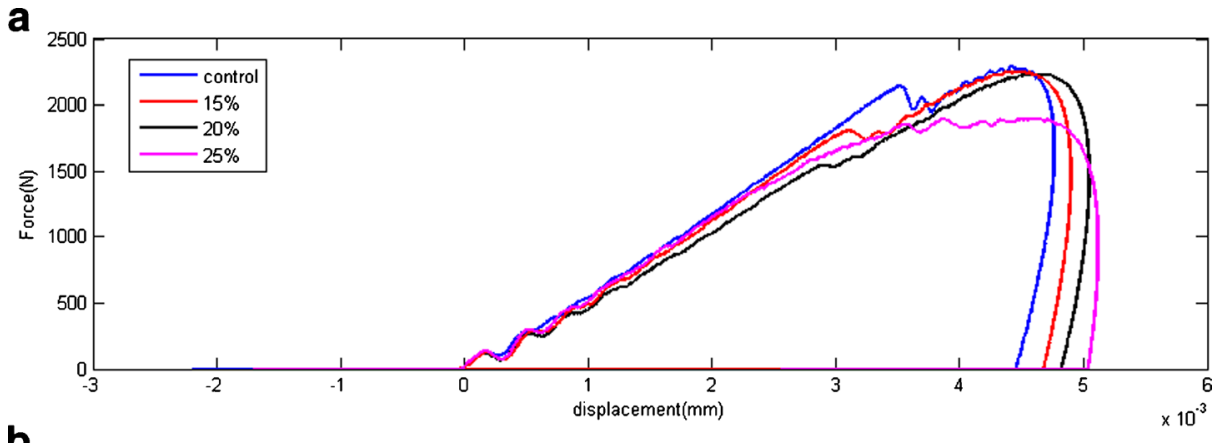

b

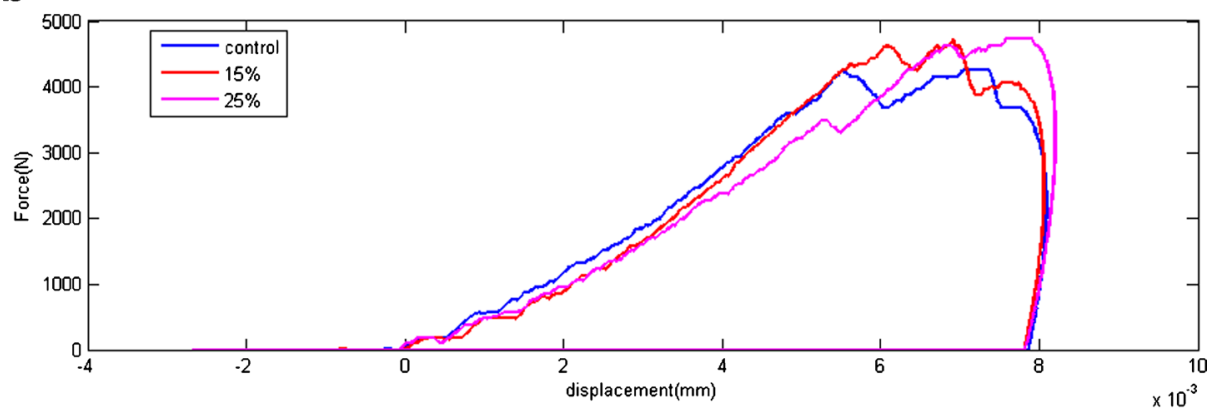

C

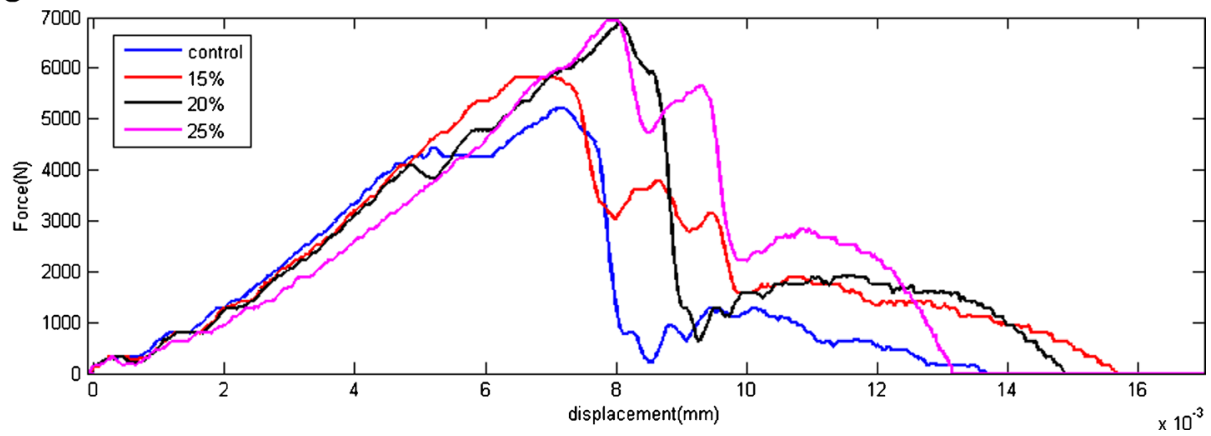

Fig. 10 Example of the force displacement curves from the impact test on $7 \mathrm{~nm}$ Silica STF reinforced CFRP: a) $6 \mathrm{~J}$; b) $20 \mathrm{~J}$ : c) $40 \mathrm{~J}$

Same trend can be observed for the energy absorption rate during the impact. In this case the effect of the percentage of $\mathrm{SiO}_{2}$ within the STF is even more evident. Indeed as it is possible to observe from Fig. 11b, while for 6 and $20 \mathrm{~J}$ impacts the trend is basically linear, when the impact velocity is raised up to $2.5 \mathrm{~m} / \mathrm{s}$ the presence of the STF increases the energy absorption rate by $38 \%$ for STF7/15 and almost $50 \%$ for STF7/25. These results confirm what observed in the previous paragraphs and they can be explained due to the dynamic behaviour of the STF, which activates the formation of the hydroclusters only when it is subjected to high shear stresses, thickening the fluid and enabling improved energy absorption mechanisms.

In order to understand the role of particles dimensions within the STF, a second series of test was undertaken using $14 \mathrm{~nm}$ silica particles for the STF reinforcement. 

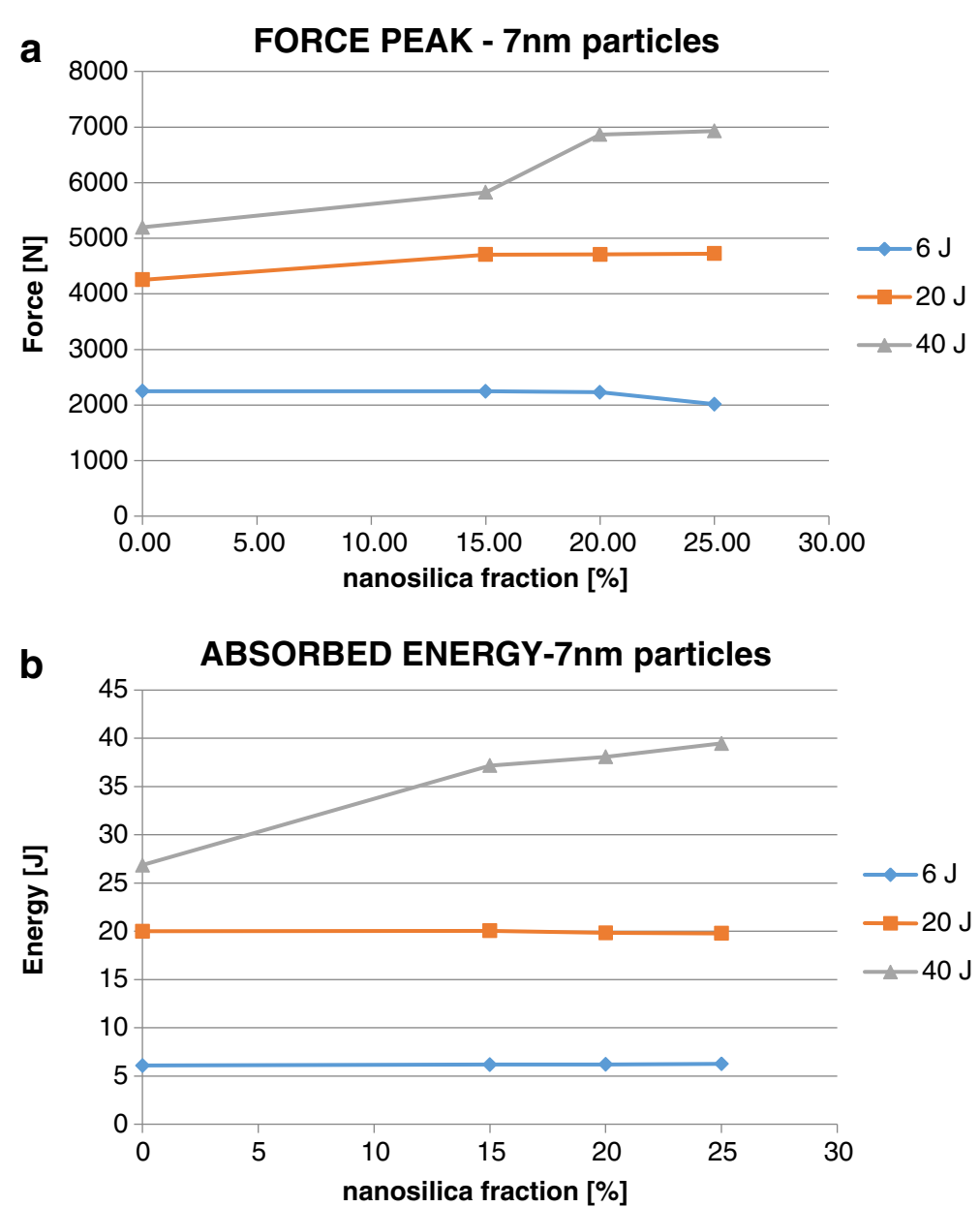

Fig. 11 Summary of the results for impact on STF7 reinforce samples: a) Force Peak variation at different energies and different silica concentrations in STF; b) Energy absorption variation at different energies and different silica concentrations in STF

Figure 12 represents the Force-Displacement curves grouped in terms of energies and in terms of percentage of $\mathrm{SiO}_{2}$ particles $(14 \mathrm{~nm})$ within the STF. As it is possible to observe from the results (Fig. 13), in this case the presence of bigger particles leads to an additional enhancement of the hybridisation effects which is particularly visible especially from the energy absorption curves. Indeed, by analysing the energy absorbed during the different impacts it is possible to observe an increase of more than $50 \%$ for the STF14/20, while for the $7 \mathrm{~nm}$ particles the percentage required in order to reach this value was $25 \%$ (STF7/20). For lower impact velocities the energy absorption levels stay basically linear as for the previous samples.

It is possible to have a clear idea of the hybridisation effects given by the presence of the STF within the CFRP by analysing Fig. 14, in which the nanoreinforced sample is compared with an unreinforced one after the impact tests. As it is possible to see from Fig. 14a and b, the solicitations generated by the $40 \mathrm{~J}$ impacts were high enough to reach critical values on the bottom lamina of the traditional laminate, resulting in a total failure of the fibres on the top 

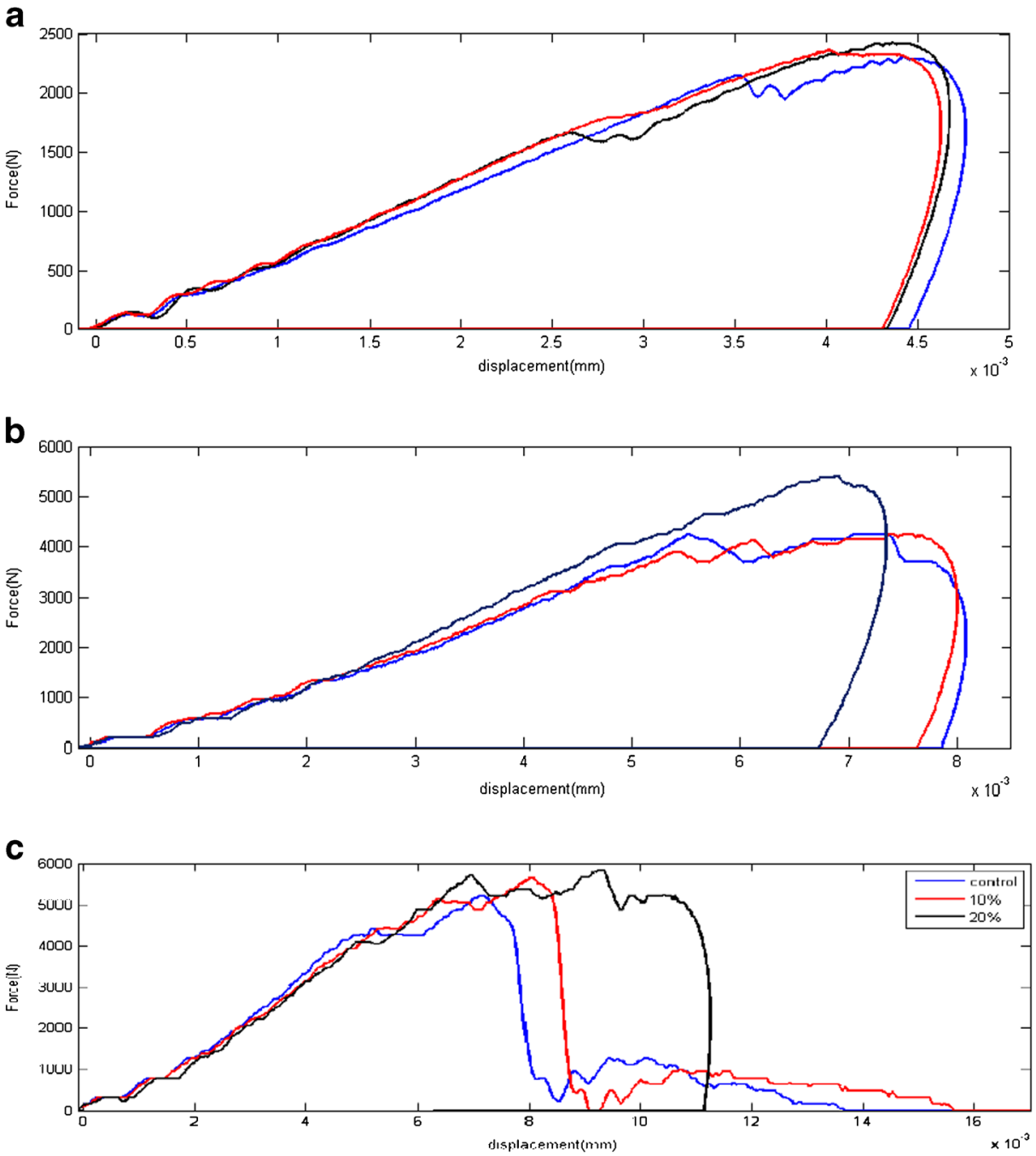

Fig. 12 Force Displacement curves from the impact test on $14 \mathrm{~nm}$ Silica STF reinforced CFRP: a 6 J; b $20 \mathrm{~J}$ : c $40 \mathrm{~J}$

surface and a complete delamination on the bottom face. On the contrary, the STF based composite kept its structural integrity after the impact showing no superficial failure on the top surface and only a small internal delamination on the bottom one (see Fig. 14a and d).

\subsubsection{C-Scan Analysis}

The internal delamination extents of the impacted samples were evaluated analysing some of the CFRP/STF samples using a C-Scan system.

$\mathrm{C}$-Scan is a non-destructive technique that uses a piezoelectric probe to send ultrasonic waves through a sample and measures the reflected signal obtained when a barrier (like a defect or the back of the sample) is reached. Analysing the difference between the signal sent 
Fig. 13 Summary of the results for impact on STF14 reinforce samples: a) Force Peak variation at different energies and different silica concentration in STF; b) Energy absorption variation at different energies and different silica concentrations in STF
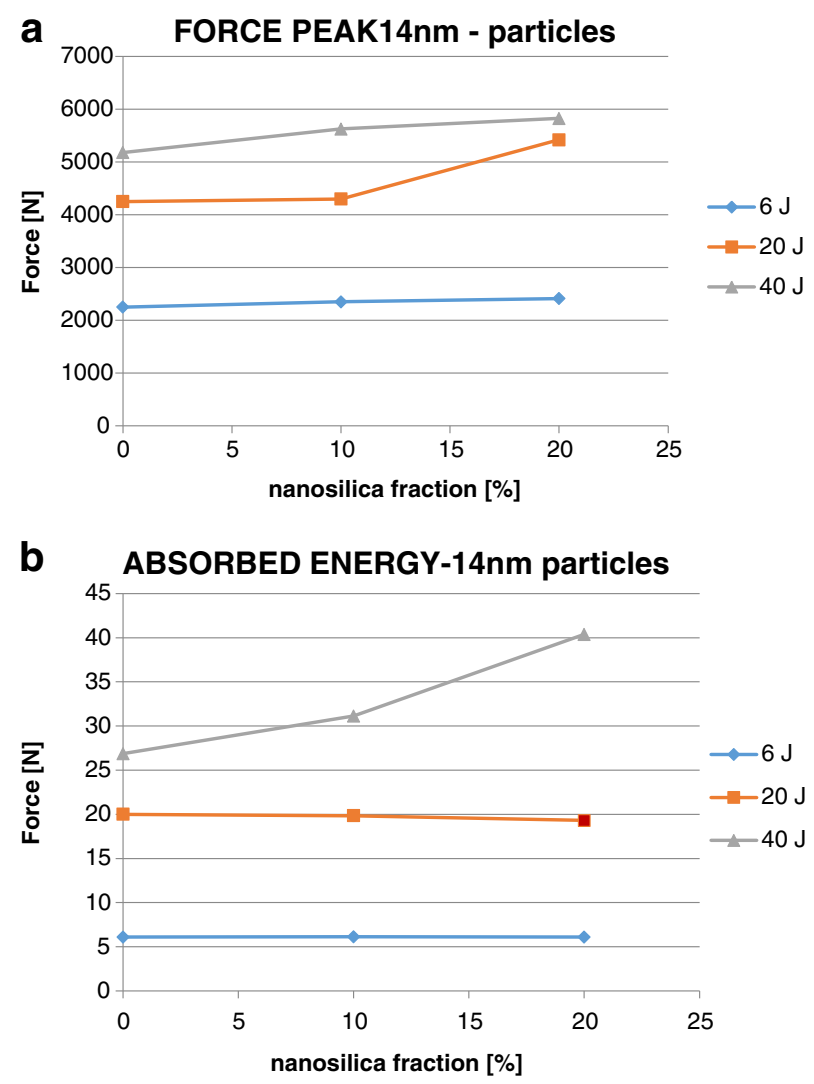

Fig. 14 Post impact image of STF reinforced sample in comparison with a traditional CFRP subjected to the same impact; a Control sample, top surface; b Control sample, bottom surface; c STF reinforced CFRP top surface; $\mathbf{d}$ STF reinforced CFRP bottom surface

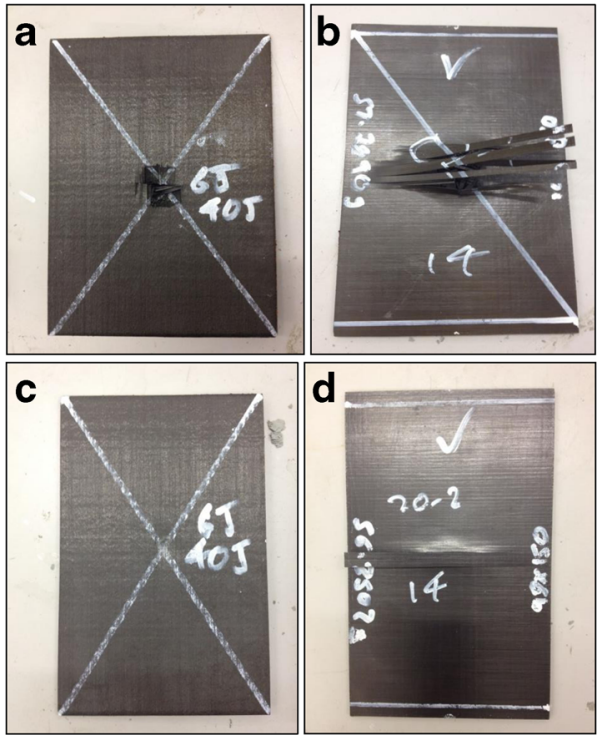


Fig. 15 C-Scan analysis of Impacted STF samples and traditional CFRP impacted at $40 \mathrm{~J}$
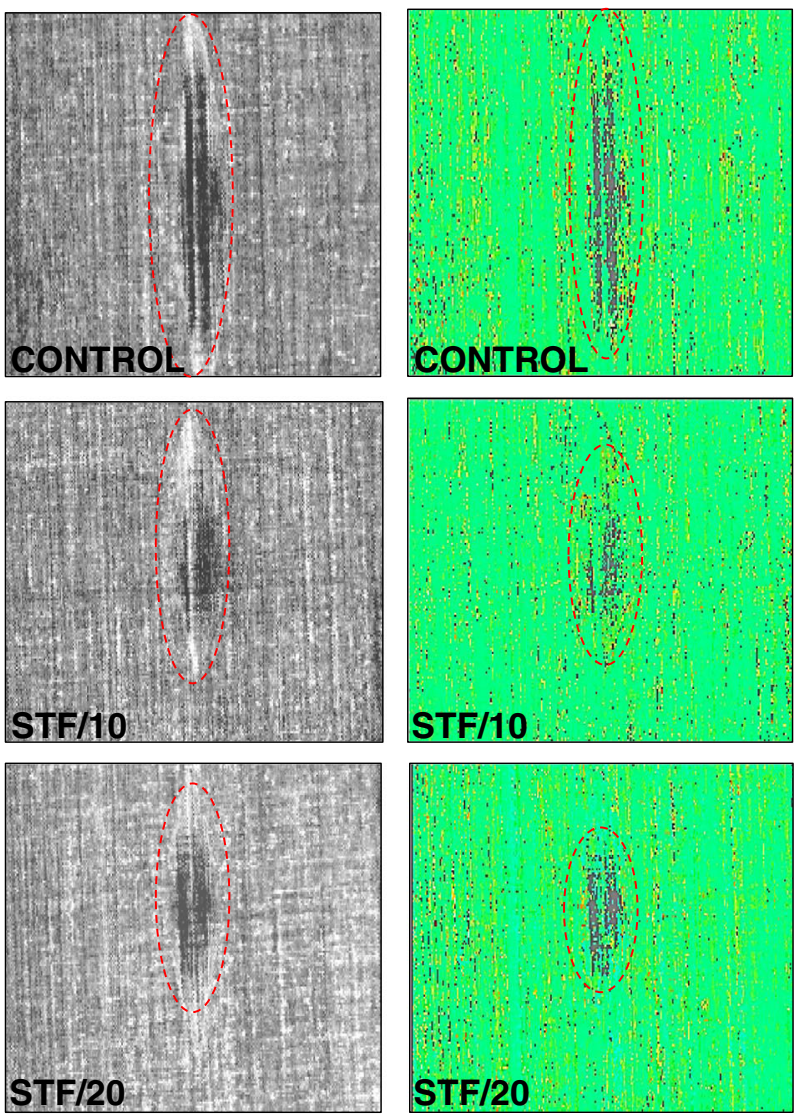

and the one collected by the detector it is possible to assess the delamination region within a laminate. Figure 15 represents several ultrasonic images of the control samples in comparison with hybridised laminates. As it is possible to observe, the presence of the nanofluid reduces the extent of the delamination by more than $30 \%$. Increasing the concentration of silica within the STF leads to an additional $10 \%$ reduction of the extension of the internal damage, confirming the results obtained with the impact tests.

\section{Conclusions}

The aim of this paper was to investigate the possibility to embed silica based Shear Thickening Fluids as shock absorber media within structural CFRP parts, in order to increase mechanical and impact properties. Several solutions were prepared using silica particles with different dimensions and mixed with Polyethylene Glycol at different concentrations. The viscosities of the solutions were analysed with rheological measurements and the results showed an evident increase of the viscosity once a critical shear rate $\gamma_{c}$ is reached. Further tests found out that increasing the concentration of particles within the solutions enhances the thickening effect, leading to higher level of final viscosity. Critical shear rate was found inversely proportional to the silica concentration within the STF. 
Composite samples were manufactured sandwiching an STF layer between traditional carbon prepregs layers and were tested in free and forced vibration in order to estimate the damping properties. Forced vibration analyses showed that the amplitudes of the natural frequencies of the hybridised samples are decreased and shifted to higher frequencies in comparison with a traditional CFRP, suggesting an increase of stiffness and damping ratio for the hybrid material. Free vibration tests confirmed the previous results showing an increase in both damping ratio and logarithmic decrement of more than $50 \%$ for the STF reinforced samples in comparison with the unreinforced laminates.

In order to understand the effect of the hybridisation on the out-of-plane properties, impact tests were conducted on samples with STF characterised by an increasing concentration of silica and with different dimensions. The results showed that for high velocity impacts (40 J) the presence of the embedded fluid within the CFRP structure is able to enhance the energy absorption rate by more than $50 \%$. This effect cannot be seen for lower velocity impacts (6 and $20 \mathrm{~J}$ ) because the hydroclusters activation mechanism requires relatively higher shear solicitations. Raising the concentration of silica or increasing the dimension of the particles within the STF leads to an increase of the energy absorption.

These results have been confirmed by C-Scan analyses, which showed how the inclusion of the STF is able to reduce by more than $30 \%$ the extent of the internal delamination for samples subjected to high velocity impacts. Increasing the concentration of silica within the STF enhances this effect.

Open Access This article is distributed under the terms of the Creative Commons Attribution 4.0 International License (http://creativecommons.org/licenses/by/4.0/), which permits unrestricted use, distribution, and reproduction in any medium, provided you give appropriate credit to the original author(s) and the source, provide a link to the Creative Commons license, and indicate if changes were made.

\section{References}

1. Laun H.M.: Rheological properties of aqueous polymer dispersions. Die. Angew. Makromol. Chem. 123(1), 335-359 (1984)

2. Hassan T.A., Rangari V.K., Jeelani S.: Synthesis, processing and characterization of shear thickening fluid (STF) impregnated fabric composites. Mater. Sci. Eng. A. 527(12), 2892-2899 (2010)

3. Lee Y.S., Wetzel E.D., Wagner N.J.: The ballistic impact characteristics of Kevlar® woven fabrics impregnated with a colloidal shear thickening fluid. J. Mater. Sci. 38(13), 2825-2833 (2003)

4. Decker M.J., Halbach C.J., Nam C.H., Wagner N.J., Wetzel E.D.: Stab resistance of shear thickening fluid (STF)-treated fabrics. Compos. Sci. Technol. 67(3-4), 565-578 (2007)

5. Lomakin E., Mossakovsky P., Bragov A., Lomunov A., Konstantinov A., Kolotnikov M., et al.: Investigation of impact resistance of multilayered woven composite barrier impregnated with the shear thickening fluid. Arch. Appl. Mech. 81(12), 2007-2020 (2011)

6. Fischer C., Braun S.A., Bourban P.E., Michaud V., Plummer C.J.G., Månson J.A.E.: Dynamic properties of sandwich structures with integrated shear-thickening fluids. Smart Mater. Struct. 15(5), 1467 (2006)

7. Soutrenon. M, Michaud. V. Structural damping using encapsulated shear thickening fluids. 83410S-S. (2012)

8. Picken, SJ, Marissen, R, Antonelli, V, Jansen, GW. Damage tolerant composite material. WO Patent WO/ 2009/142, 491 (2009)

9. Liu Y., Wang Z., Zhang X.: Characterization of supramolecular polymers. Chem. Soc. Rev. 41(18), 59225932 (2012)

10. Wagner N.J., Brady J.F.: Shear thickening in colloidal dispersions. Phys. Today. 62(10), 27-32 (2009)

11. Kaldasch J., Senge B., Laven J.: Shear thickening in concentrated soft sphere colloidal suspensions: a shear induced phase transition. Journal of Thermodynamics. 2015, 10 (2015)

12. Bender J.W., Wagner N.J.: Optical measurement of the contributions of colloidal forces to the rheology of concentrated suspensions. J. Colloid. Interface. Sci. 172(1), 171-184 (1995) 
13. Maranzano B.J., Wagner N.J.: Flow-small angle neutron scattering measurements of colloidal dispersion microstructure evolution through the shear thickening transition. J. Chem. Phys. 117(22), 10291-10302 (2002)

14. Maranzano B.J., Wagner N.J.: The effects of particle size on reversible shear thickening of concentrated colloidal dispersions. J. Chem. Phys. 114(23), 10514-10527 (2001)

15. Xianzhou Z., Weihua L., Gong X.L.: Study on magnetorheological shear thickening fluid. Smart. Mater. Struct. 17(1), (2008)

16. Lee Y.S., Wagner N.J.: Rheological properties and small-angle neutron scattering of a shear thickening, nanoparticle dispersion at high shear rates. Ind. Eng. Chem. Res. 45(21), 7015-7024 (2006)

17. Brown E., Forman N.A., Orellana C.S., Zhang H., Maynor B.W., Betts D.E., et al.: Generality of shear thickening in dense suspensions. Nat. Mater. 9(3), 220-224 (2010)

18. Masoudi A., Madaah Hosseini H.R., Shokrgozar M.A., Ahmadi R., Oghabian M.A.: The effect of poly(ethylene glycol) coating on colloidal stability of superparamagnetic iron oxide nanoparticles as potential MRI contrast agent. Int. J. Pharm. 433(1-2), 129-141 (2012)

19. Jiang W., Sun Y., Xu Y., Peng C., Gong X., Zhang Z.: Shear-thickening behavior of polymethylmethacrylate particles suspensions in glycerine-water mixtures. Rheol. Acta. 49(11-12), 1157-1163 (2010)

20. Wetzel E.D., Lee Y.S., Egres R.G., Kirkwood K.M., Kirkwood J.E., Wagner N.J.: The Effect of Rheological Parameters on the Ballistic Properties of Shear Thickening Fluid (STF)-Kevlar Composites. AIP. Conf. Proc. 712(1), 288-293 (2004)

21. Peng S.T.J., Landel R.F.: Rheological behavior of progressively shear thickening solutions. J. Appl. Phys. 52(10), 5988-5993 (1981)

22. Choi G.N., Krieger I.M.: Rheological studies on sterically stabilized model dispersions of uniform colloidal spheres: II. Steady-shear viscosity. J. Colloid. Interface. Sci. 113(1), 101-113 (1986)

23. Boersma W.H., Laven, J., Stein H.N.: Shear thickening (dilatancy) in concentrated dispersions. AICHE. J. 36(3), 321-332 (1990)

24. Stickel J.J., Powell R.L.: Fluid mechanics and rheology of dense suspensions. Annu. Rev. Fluid Mech. 37, 129-149 (2005)

25. Kang T., Hong K., Yoo M.: Preparation and properties of fumed silica/Kevlar composite fabrics for application of stab resistant material. Fibers. Polym. 11(5), 719-724 (2010)

26. Rao SS, Yap FF. Mechanical Vibrations: Addison-Wesley New York; (1995)

27. YP L., Killian J.W., Everstine G.C.: Vibrations of three layered damped sandwich plate composites. J. Sound. Vib. 64(1), 63-71 (1979) 